\title{
A fresh look at the free flight dynamics of a spinning projectile
}

\author{
SMITA B BAKSHI and K C SHARMA \\ Institute of Armament Technology, Pune 411 025, India \\ MS received 1 September 1984; revised 3 February 1987
}

\begin{abstract}
In the first part of the paper, several equations of motion by different groups of workers are critically examined. Exact equations of motion are then developed. This form is then quasi-linearised into a ballistic formulation. The geometrical motion of a spinning projectile, in the presence of a cubic restoring moment is analysed in the cross-flow plane. It is observed that the trajectories assume a cuspidal form at the lower and upper range of the travel, when the angular momentum parameter coincides with these points. In general, both steady and unsteady precessional motions are presented.
\end{abstract}

Keywords. Spinning projectile; precession; cross-flow plane; pseudo stability frame.

\section{Introduction}

We present this work in two parts. In the first $(\S 2)$, the equations of motion of a spinning projectile with six degrees of freedom are formulated. The second part $(\$ 3)$ deals with the geometrical presentation of the projectile motion in the cross-flow plane.

Without going into a lengthy historical discussion, most of the recent work in motion analysis of a free flight projectile has been based on the models suggested by Fowler et al (1920), Murphy (1963) and Nielson \& Synge (1946) (NS hereafter).

These models are developed for one or the other consideration of flight dynamics of projectiles but do not depict the complete story of reference frames and aerodynamic forces. An attempt has been made in this work to analyse these models and present a complete set of motion equations for the free flight motion of a symmetric spinning projectile, referred to hereafter only as projectile.

The oscillatory motion of the projectile based on the work of Fowler et al (1920) and Murphy (1963) leads to the presentation of this motion over the surface of the sphere, an analogy which has been extended from the well-analysed motion of a spinning top. The oscillatory motion in fact occurs only over the cross-flow plane of the projectile. Part two of the work analyses the different geometrical trajectories of motion in this plane. 


\section{The equations of motion}

Given the projectile characteristics, the equations of motion can be well-defined, if one correctly selects the reference frame wherein the trajectory variables $(\bar{V}, \bar{H}, \bar{\omega}, \vec{\Omega})$, and the forces of gravity and aerodynamics can be presented in a simple way. Also, the equations should be such that the forces of gravity and aerodynamics can be treated for a nonlinear motion of the projectile with higher accuracy. However, the equations may be reducible to the models obeying the principles of linearity (quasilinearity) and/or the slow variability along the flight path.

First, we discuss the models of Fowler et al (1920), Murphy (1963) and Nielson \& Synge (1946).

\subsection{Fowler model}

Wind axes are employed for oscillatory motion and are connected to the fixed earth axis system. The projectile axes then are defined relative to the wind axes. The $\gamma$-type equations are derived on the following assumptions.

(i) The centre of gravity of the projectile moves in a horizontal straight line uniformly. This means that the projectile, for theoretical purposes, is fired horizontally with a large muzzle velocity.

(ii) The curvature due to gravity is neglected.

(iii) There is no decay of the total axial spin.

NS have discussed the following inconsistencies of this model.

(i) Their aerodynamic force system is not the most general system consistent with (a) the aerodynamic hypothesis, (b) linear dependence on the cross components in the case of small yaw, and (c) the symmetry of the shell.

(ii) The system does not satisfy the fundamental requirement of invariance with respect to shift of mass centre. In addition, the derived equations lack further consistency on the following counts.

(i) The effect of the motion of the wind axes relative to earth has been altogether ignored.

(ii) The wind axes cannot be recognised for measurement purposes.

\subsection{BRL Model}

Murphy has discussed two different exact models of the equations of motion in his research work in the BRL reports (Murphy 1953, 1963). One model (Murphy 1953) of equations is that of NS, which we discuss later. We analyse one of the reports (Murphy 1963) in this section. Murphy (1963) assumes a non-rolling body axes system for presenting his equations of motion. Non-rolling body axes cannot be recognised for experimental work, therefore any analysis would require intermediary transformations to match the analytical results to the experimental data. Also, the definition, $\xi=(v+i w) / V$, introduces, in the equations, another term, $\gamma=(u / V)$, and its derivative. Since the relation $\gamma^{2}=1-|\xi|^{2}$ holds, the presence of $\gamma$ and its derivative induces nonlinearity in $\xi^{\prime}$, in the equations of motion, which could be otherwise linear. We refer to the term $\left(\gamma^{\prime} / \gamma\right)$ in (IV.6.8) (Murphy 1963), and the assumptions in $\gamma$ (VI.6.9) are, therefore, additional restrictions for linearisation. 
Finally, the aerodynamic coefficients expressed in the Prandtl notation require a highly simplified assumption $\alpha_{r}^{2}=\alpha^{2}+\beta^{2}$. This simplification is acceptable in aircraft and control missile dynamics, but cannot be used for a free flight body with a long range and large time of flight. Further this assumption cannot be acceptable for high angle flight dynamics.

Etkin (1971, pp. 116-117) defines angle of attack and sideslip as $\alpha=\tan ^{-1} w / u$, $\beta=\sin ^{-1} v / V$ in (IV.5.5). From the above, we obtain

$$
\begin{aligned}
& \mathrm{d} \alpha=\left\{1 /\left[1+(w / u)^{2}\right]\right\}\left[(\mathrm{d} w / u)-\left(w \mathrm{~d} u / u^{2}\right)\right], \\
& \mathrm{d} \beta=\left\{1 /\left[1-(v / V)^{2}\right]\right\}\left[(\mathrm{d} v / V)-\left(v \mathrm{~d} V / V^{2}\right)\right] .
\end{aligned}
$$

These differentials, on applying the small angle theory, simplify to

$$
\begin{aligned}
& \mathrm{d} \alpha=\mathrm{d} w / u, \\
& \mathrm{~d} \beta=\mathrm{d} u / v .
\end{aligned}
$$

This simplification has been successfully applied for analysis in aircraft and control missile dynamics. We assert that this simplification raises the following inconsistencies.

(i) The non-dimensionalisation, in the above simplification, has turned out to be different, namely, in $\mathrm{d} \alpha$ it is with respect to $u$ and in $\mathrm{d} \beta$ it is with respect to $V$. It will further transmit $\gamma(=u / v)$ terms in the equations of motion as one moves from the wind axes to the body axes system.

(ii) The aerodynamic nonlinearities in Prandtl notation are introduced through $\alpha^{2}$, $\beta^{2}$ etc. The force functions are derived in the body axes system (Maple \& Synge 1949), since the aerodynamic force is a body force. A transformation from body axes to wind axes will involve trigonometrical functions in $\alpha$ and $\beta$. The approximation to separable power series in $\alpha$ and $\beta$ is an over-simplification.

From the above, it is concluded that the aerodynamic force system is to be represented as velocity in body axes frame.

\subsection{NS model}

NS have approached the formulation of the ballistic six-degrees-of-freedom equations in an exact manner. The choice of the reference frame takes care of the experimentation, as well as the mathematical simplicity and correctness. The aerodynamic forces (Maple \& Synge 1949) are derived in the body axes. Thus a transformation is needed to express this force system in the pseudo frame of reference. This aspect has not been critically examined by NS in their work. Second, the analysis has been made in the time variable and this places restrictions on the outcome. The effect of the variation of axial velocity on the angular motion has been suppressed. Third, the work does not refer to the equivalence of the angular momentum in two frames. This has been examined by Murphy (1963).

The work of Murphy (1953) has been presented on this pattern along with the transformation of time variable to a space variable.

\subsection{Exact equations with six degrees of freedom}

The Newtonian equations of motion are expressed in the pseudo stability (PS) frame 


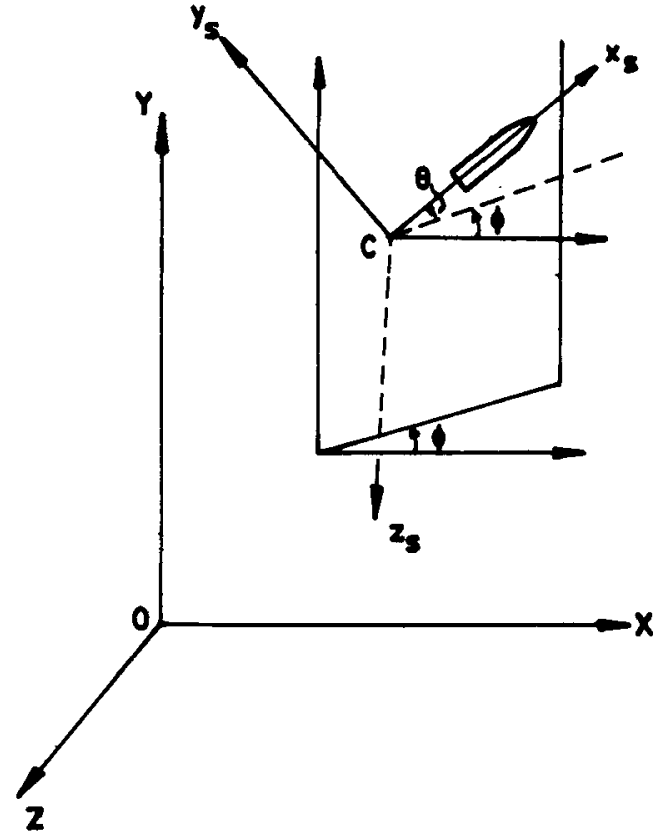

Figure 1. Pseudo stability frame of a projectile.

of NS (figure 1). The vertical plane contains the projectile axis $C X_{s}$. The normal to this is directed along $C Z_{s}$. The vertical plane subtends an angle $\phi$ with the $X O Y$ plane of the earth axes. The axis $C X_{s}$ is inclined to the horizontal at an angle $\theta$. The transformation matrix relation is

$$
\left[\begin{array}{l}
\hat{I} \\
\hat{J} \\
\hat{R}
\end{array}\right]=\left[\begin{array}{ccl}
\cos \theta \cos \phi & -\sin \theta & \cos \phi \sin \phi \\
\sin \theta & \cos \theta & 0 \\
-\cos \theta \sin \phi & \sin \theta & \sin \phi \cos \phi
\end{array}\right]\left[\begin{array}{l}
\hat{i}_{s} \\
\hat{j}_{s} \\
\hat{k}_{s}
\end{array}\right]
$$

The angular velocity $\overline{\boldsymbol{\Omega}}$ of this frame is given by

$$
\bar{\Omega}=\dot{\phi} \sin \theta \hat{i_{s}}+\dot{\phi} \cos \theta \hat{j_{s}}+\theta \hat{k_{s}} \text {. }
$$

If a roll rotation $\psi$ is made with respect to the PS axes, one obtains the body axes of the projectile. The angular velocity $\bar{\omega}$ of the projectile will work out to be

$$
\bar{\omega}(p, q, r)=(\dot{\psi}+\dot{\phi} \sin \theta) \hat{i_{b}}+\dot{\phi} \cos \theta \hat{j}_{s}+\dot{\theta} \hat{k}_{s} \text {. }
$$

The transformation matrix relating PS axes to body axes is

$$
\left[\begin{array}{c}
\hat{i}_{b} \\
\hat{j}_{b} \\
\hat{k}_{b}
\end{array}\right]=\left[\begin{array}{ccl}
1 & 0 & 0 \\
0 & \cos \psi & \sin \psi \\
0 & -\sin \psi & \cos \psi
\end{array}\right]\left[\begin{array}{c}
\hat{i}_{s} \\
\hat{j}_{s} \\
\hat{k}_{s}
\end{array}\right] .
$$

The angular momentum vector $H\left(I_{x x} p_{b}, I_{y y} q_{b}, I_{y y} r_{b}\right), I_{z z}=I_{y y}$ for rotationally symmetric projectiles of order three and more, in the PS system is

$$
H=I_{x x} p_{b} \hat{\hat{i}_{b}}+I_{y y} q_{b} \hat{\hat{j}_{b}}+I_{y y} r_{b} \hat{k}_{b},
$$




$$
\begin{aligned}
\bar{H}= & I_{x x} p_{s} \hat{i_{s}}+I_{y y}\left[q_{b}\left(\cos \psi \hat{j_{s}}+\sin \psi \hat{k_{s}}\right)+\right. \\
& \left.+r_{b}\left(-\sin \psi \hat{j_{s}}+\cos \psi \hat{k_{s}}\right)\right] \\
= & I_{x x} p_{s} \hat{\hat{i}_{s}}+I_{y y}\left[\left(q_{b} \cos \psi-\sin \psi r_{b}\right) \hat{\hat{j}_{s}}+\right. \\
& \left.+\left(q_{b} \sin \psi+r_{b} \cos \psi\right) \hat{k_{s}}\right] \\
= & I_{x x} p_{s} \hat{i_{s}}+I_{y y}\left(q_{s} \hat{j_{s}}+r_{s} \hat{k}_{s}\right) .
\end{aligned}
$$

The aerodynamic force $\overline{\mathscr{F}}\left(\mathscr{F}_{x}, \mathscr{F}_{y}, \mathscr{F}_{z}\right)$ and moment $\overline{\mathscr{G}}\left(\mathscr{S}_{x}, \mathscr{G}_{y}, \mathscr{G}_{z}\right)$ with these components in body frame and the velocities $\bar{V}$ and $\bar{\omega}$ transform as follows.

$$
\begin{aligned}
& u_{b}=u_{s}, \\
& \xi_{b}=\xi_{s} \exp (-i \psi), \\
& \eta_{b}=\eta_{s} \exp (-i \psi), \\
& p_{b}=p_{s}, \\
& \mathscr{F}_{x b}=\mathscr{F}_{x s}, \\
& F_{b}=F_{s} \exp (-i \psi), \\
& \mathscr{G}_{x b}=\mathscr{G}_{x s}, \\
& G_{b}=G_{s} \exp (-i \psi),
\end{aligned}
$$

where

$$
\begin{aligned}
& \xi=v+i w / u, \\
& \eta=(q+i r) l / u, \\
& F=F_{y}+i \mathscr{F}_{z}, \\
& G=\mathscr{G}_{y}+i \mathscr{G}_{z}, \\
& \psi=\int_{0}^{i}\left(p_{s}-\Omega_{x}^{s}\right) \mathrm{d} t,
\end{aligned}
$$

and $l$ is defined as the characteristic length of the projectile. Since the projectile axis also points along the $x_{s}$ axis, we have

$$
\bar{\Omega} \Lambda \hat{i}_{s}=\bar{\omega} \Lambda \hat{i}_{b},
$$

resulting in $q_{s}=\Omega_{y}^{s}, \quad r_{s}=\Omega_{z}^{s}$. The components of gravitational acceleration $\bar{g}$ in PS are

$$
\begin{aligned}
& g_{x}^{s}=-g \sin \theta, \\
& g_{y}^{s}=-g \cos \theta, \\
& g_{z}^{s}=0
\end{aligned}
$$

The exact Newtonian equations of motion,

$$
\begin{aligned}
& (\delta / \mathrm{d} t) \bar{V}_{s}+\bar{\Omega} \Lambda \bar{V}_{s}=\bar{g}_{s}+\left(\overline{\mathscr{F}}_{s} / m\right), \\
& (\delta / \mathrm{d} t) \vec{H}_{s}+\bar{\Omega} \Lambda \tilde{H}_{s}=\overline{\mathscr{G}}_{s},
\end{aligned}
$$

where $(\delta / \mathrm{d} t)$ is the relative differentation in the PS frame, in complex form are

$$
\begin{aligned}
& \dot{u}_{s}+q_{s} w_{s}-r_{s} v_{s}=g_{x}^{s}+\left(F_{x s} / m\right), \\
& \dot{\xi}_{s}+\xi_{s}\left[\left(\dot{u}_{s} / u_{s}\right)+i \Omega_{x}^{s}\right]-\left(i u_{s} \eta_{s} / l\right)=\left[g_{y}^{s}+\left(F_{s} / m\right)\right]\left(l / u_{s}\right), \\
& \dot{\eta}_{s}+\left\{\left(\dot{u}_{s} / u_{s}\right)-i\left[\left(B^{\prime} \zeta_{s} u_{s} / l\right)-\Omega_{x}^{s}\right]\right\} \eta_{s}=\left(G_{s} l\right) /\left(u_{s} I_{y y}\right), \\
& \dot{\zeta}_{s}+\left(\dot{u}_{s} / u_{s}\right) \zeta_{s}=\left(\mathscr{G}_{x} l\right) /\left(I_{x x} u_{s}\right),
\end{aligned}
$$

where $B^{\prime}=I_{x x} / I_{y y}, \quad \zeta=p l / u$ (non-dimensional spin) and $g_{z}^{s}=0$ from (5). 


\subsection{Ballistic form of exact equations}

It is assumed that the term $\left(q_{s} w_{s}-r_{s} v_{s}\right)=o\left(\dot{u}_{s}\right)$ is ignored in the forward motion of the projectile given by (7a). Secondly the equations are expressed in the nondimensional axial distance $\bar{x}=\left(x_{s} / l\right)$, through the transformation

$$
\mathrm{d} / \mathrm{d} t=\left(u_{s} / l\right)(\mathrm{d} / \mathrm{d} \bar{x}) \text {. }
$$

Equations (6) in scalar form now are,

$$
\begin{aligned}
& u_{s}^{\prime}=\left(g_{x}^{s} l / u_{s}\right)+\left(F_{x}^{s} l / u_{s} m\right) \\
& \xi_{s}^{\prime}+\left[\left(g_{x}^{s} l / u_{s}^{2}\right)+\left(F_{x}^{s} l / u_{s}^{2} m\right)+i \bar{\Omega}_{x}^{s}\right] \quad \xi_{s}-i \eta_{s}=\left[\left(g_{y}^{s} l / u_{s}^{2}\right)+\left(F_{s} l / u_{s}^{2} m\right)\right] \\
& \eta_{s}^{\prime}+\left[\left(g_{x}^{s} l / u_{s}^{2}\right)+\left(F_{x}^{s} l / u_{s}^{2} m\right)-i\left(B^{\prime} \zeta_{s}-\bar{\Omega}_{x}^{s}\right)\right] \eta_{s}=\left(G_{s} l^{2}\right) /\left(u_{s}^{2} I_{y y}\right) \\
& \zeta_{s}^{\prime}+\left[\left(g_{x}^{s} l / u_{s}^{2}\right)+\left(F_{x}^{s} l / u_{s}^{2} m\right)\right] \zeta_{s}=\left(\mathscr{G}_{x}^{s} l^{2}\right) /\left(u_{s}^{2} I_{x x}\right),
\end{aligned}
$$

where

$$
\overline{\mathbf{\Omega}}_{x}=\Omega_{x}^{s} l / u_{s} ; I^{\prime}=\mathrm{d} / \mathrm{d} \bar{x}, \quad K_{t}^{2}=I_{y y} / m l^{2} .
$$

From the above ballistic equations, a second order differential equation in $\xi_{s}$ is derived by eliminating $\eta_{s}$ (where possible) between (8b) and (8c) to obtain different mathematical models for various situations. The equation in $\xi_{s}$ is of the form

$$
\xi_{s}^{\prime \prime}+\left(K_{1}-i K_{2}\right) \xi_{s}^{\prime}+\left(K_{3}-i K_{4}\right) \xi_{s}=K_{5}+i K_{6} .
$$

Here $K_{i}(i=1,2,3,4)$ refer to damping, spin, positional and Magnus (non-positional) parameters. These parameters are functions of the density of air, aerodynamic coefficients, spin $\zeta_{s}$, frame velocity $\bar{\Omega}_{x}^{s}$, non-dimensional gravity groups $\left(g_{x}^{s} l / u_{s}^{2}\right)$, $\left(g_{y}^{s} l / u_{s}^{2}\right)$ for linear force systems. However the $K^{\prime}$ will be functions of $\xi_{s}$ for nonlinear motion.

\section{Projectile motion in the cross-flow plane}

The intent of this section is to depict the geometrical trajectories in the cross-flow plane. It is assumed that the cross torque due to cross velocity (restoring moment) is cubic in cross velocity. The aerodynamic forces appear due to the translatory motion only. Thus the force system is

$$
\begin{aligned}
& \mathscr{F}_{x}^{s}=-\frac{1}{2} \rho u_{s}^{2} S C_{x}, \\
& F_{s}=-\frac{1}{2} \rho u_{s}^{2} S f_{1} \xi_{s}, \\
& G_{s}=-\frac{1}{2} i \rho u_{s}^{2} S l\left(g_{1}+g_{3} \xi_{s} \bar{\xi}_{s}\right) \xi_{s}, \\
& \mathscr{S}_{x}^{s}=0 .
\end{aligned}
$$

It is further assumed that the effect of forward motion on axial spin $\zeta_{s}$ is negligible, i.e. $\left[\left(g_{x}^{s} l / u_{s}^{2}\right)-\varepsilon C_{x}\right] \zeta_{s}=o\left(\zeta_{s}^{\prime}\right)$. This results in the constancy of axial spin $\left(\zeta_{s}\right)=\left(\zeta_{s}^{0}\right)$ over the flight of the projectile. Further it is also assumed that the coefficients of $\zeta_{s}$ and $\eta_{s}$ are nonvarying in (8b) and (8c). The $K$ 's in (10) then are

$$
\begin{aligned}
& K_{1}=-2 \varepsilon C_{x}+\left(2 g_{x} l / u_{s}^{2}\right), \\
& K_{2}=B^{\prime} \zeta_{s}^{0}-2 \bar{\Omega}_{x}^{s},
\end{aligned}
$$




$$
\begin{aligned}
K_{3}= & -\varepsilon K_{t}^{-2}\left(g_{1}+g_{3} \xi_{s} \bar{\xi}_{s}\right)+\varepsilon^{2}\left(C_{x}-f_{1}\right) C_{x}+\left(g_{x}^{s} l^{2} / u_{s}^{2}\right)- \\
& -\bar{\Omega}_{x}^{2}-\left(g_{x}^{s} l / u_{s}^{2}\right)\left(2 C_{x}-f_{1}\right)+B^{\prime} \zeta_{s}^{0} \bar{\Omega}_{x}^{s}, \\
K_{4}= & -B^{\prime} G_{s}^{0}\left(C_{\bar{x}}-f_{1}\right)+B^{\prime} \zeta_{s}^{0}\left(g_{x}^{s} l / u_{s}^{2}\right)+\bar{\Omega}_{x}^{s}\left[\varepsilon\left(2 C_{x}-f_{1}\right)-\right. \\
& \left.-\left(2 g_{x}^{s} l / u_{s}^{2}\right)\right], \\
K_{5}= & -\left[\varepsilon C_{x}-\left(g_{x}^{s} l / u_{s}^{2}\right)\right]\left(g_{y}^{s} l / u_{s}^{2}\right), \\
K_{6}= & \left(\bar{\Omega}_{x}^{s}-\zeta_{s}^{0} B^{\prime}\right)\left(g_{y}^{s} l / u_{s}^{2}\right) .
\end{aligned}
$$

We consider the undamped natural motion with non-Magnus character for the geometrical behaviour. We also split $K_{3}$ into its linear and nonlinear part

$$
K_{3}=K_{30}+K_{31} \xi_{s} \bar{\xi}_{s} \text {. }
$$

The equation under consideration is

$$
\xi_{s}^{\prime \prime}-i K_{2} \xi_{s}^{\prime}+\left(K_{30}+K_{31} \xi_{s} \bar{\xi}_{s}\right) \xi_{s}=0 .
$$

The complex number $\xi$, recalling that $\xi=(v+i w) / u$, represents the plane at a unit distance from the pole $C$ (origin of the PS coordinates) of the projectile along its axis (see figure 2).

The motion given by $\xi_{s}$ in the plane, thus refers to the velocity vector relative to the shell axis. In the substitution

$$
\xi_{s}=\delta e^{i \phi}
$$

$\delta=\tan v,[(-\pi / 2 \leqslant v \leqslant \pi / 2)$ and $v$ is the total angle of attack $]$. Further if we write

$$
Z=\delta^{2} \text {, }
$$

the first integrals of (12) are

$$
\begin{aligned}
& \phi^{\prime}=\left(K_{2} / 2\right) G(Z), \\
& Z^{\prime 2}=K_{2}^{2} \sigma^{2} H(Z), \quad(0 \leqslant Z<\infty),
\end{aligned}
$$

where

$$
G(Z)=\left(Z-Z_{*}\right) / Z,
$$

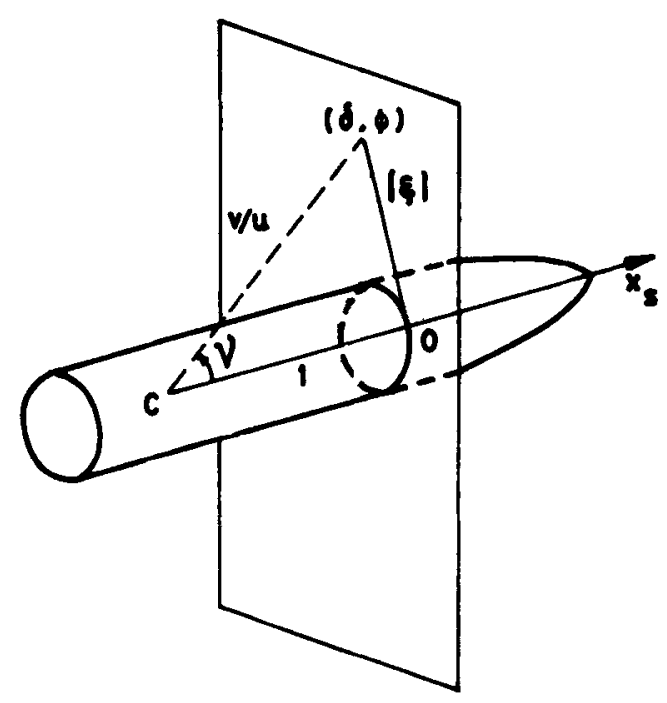

Figure 2. Cross-flow plane of a projectile. 


$$
\begin{aligned}
H(Z) & =-m Z^{3}-Z^{2}+4 E Z-4 F^{2} \\
Z_{*} & =-2 F \sigma \quad\left(-\infty<Z_{*}<\infty\right) \\
\sigma^{2} & =1+\left(4 K_{30} / K_{2}^{2}\right) \quad(\text { Cranz stability parameter }) \\
m & =2 K_{31} /\left(K_{2}^{2} \sigma^{2}\right) .
\end{aligned}
$$

Here $E$ and $F$ are energy and angular momentum constants of motion defined suitably.

One may map the $\xi$ plane over the sphere through $t=1 /(1+Z)$ to discuss the motion.

To consider the geometrical (Routhian) motion, the general values of $E$ and $F$ are considered arising out of non-zero positions $\left(\delta_{0}, \phi_{0}\right)$ and velocities $\left(\delta_{0}^{\prime}, \phi_{0}^{\prime}\right)$. We state, without proof, the following particular cases.

Corollary $I$ : For $E=F$ and $m>0$, the motion commences from $Z_{0}=0, Z_{0}^{\prime}=0, \phi_{0}^{\prime}$ is arbitrary.

Corollary $I I$ : For $E=F$ and $m<0$ (i) the surface of initial conditions is symmetric about the planes $Z_{0}^{\prime}=0$ and $\phi_{0}^{\prime}=K_{2} / 2$, (ii) the projection over (a) $Z_{0}^{\prime}=0$ is a parabola, (b) for $\phi_{0}^{\prime}=K_{2} / 2$, it is a cubic curve $Z_{0}^{\prime 2}+m Z_{0}^{3}=0$, (c) for $Z_{0}=0, Z_{0}^{\prime}=0$, $\phi_{0}^{\prime}$ is arbitrary.

Corollary III: For $E(E>0)=m$, the surface of initial conditions is open and its projection over $Z_{0}^{\prime}=0$ is either a straight line $Z_{0}=0$ or an open curve symmetric about $Z_{0}$.

Corollary IV: For $F=m$, the initial conditions $\left(Z_{0}, \phi_{0}^{\prime}\right)$ lie over a rectangular hyperbola.

Corollary $V$ : For $F=0$, either the motion commences from a zero angle of attack or the procession $\phi^{\prime}$ remains $K_{2} / 2$ throughout the motion.

The roots $\left(Z_{1}, Z_{2}, Z_{3}\right)$,

$$
\begin{aligned}
& Z_{1}+Z_{2}+Z_{3}=-1 / m \\
& Z_{1} Z_{2}+Z_{2} Z_{3}+Z_{3} Z_{1}=-4 E / m \\
& Z_{1} Z_{2} Z_{3}=-4 F^{2} / m
\end{aligned}
$$

of the cubic $H(Z)$ in (16) lie as

$$
Z_{1}<0<Z_{2} \leqslant Z \leqslant Z_{3}, \quad m>0
$$

and

$$
0 \leqslant Z_{1} \leqslant Z \leqslant Z_{2} \leqslant Z_{3}, \quad m<0,
$$

for real motion (figures 3a, b).

It can also be seen that $E>0$ for real moticn. We state without proof the following cases of interest to establish the relation between these roots.

Corollary VI: For any of the cases $E=F, F=m, E=m$ the roots of the cubic $H(Z)$ lie over an open surface. In the case of $E=F$ only, the surface passes through the origin. Corollary VII: For $E=m$, the projections over the coordinate planes are rectangular hyperbolas. 

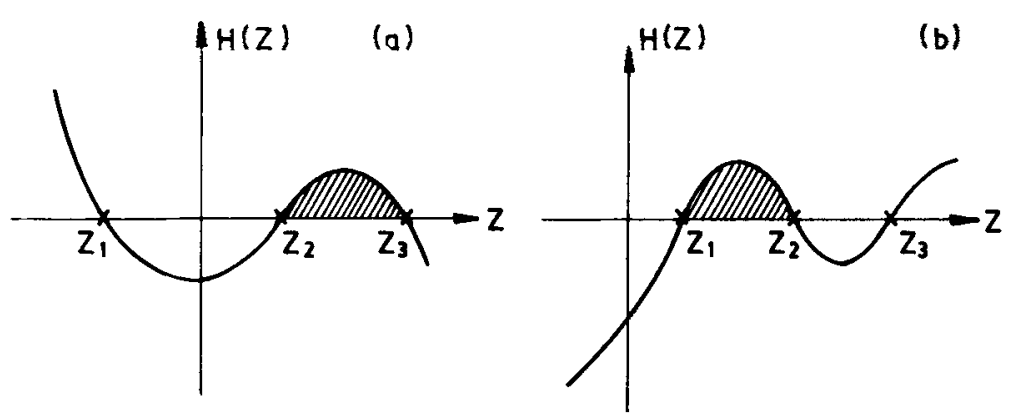

Figure 3. Location of roots of $H(z)$ for real motion for (a) $m>0$, (b) $m<0$.

\subsection{Circles of no velocity}

The velocity $\bar{V}\left(V_{r}=\delta^{\prime}, V_{T}=\delta \phi^{\prime}\right)$ of the point $(\delta, \phi)$ in the total angle of attack plane is given by

$$
V^{2}=\left(K_{2}^{2} \sigma^{2} / 4\right) \psi(Z)
$$

where

$$
\psi(Z)=-m Z^{2}+\left[\left(1 / \sigma^{2}\right)-1\right] Z+(4 F / \sigma)+4 E .
$$

The circles of no velocity $(V=0)$ are the set of points $[G(Z)=0] \bigcap[H(Z)=0]$. We obtain that $Z=Z_{*}$ is a circle of no velocity if it is a zero of $H(Z)$. The curves $\psi(Z)$ are shown in figures 4,5 and 6 for circles of no velocity.
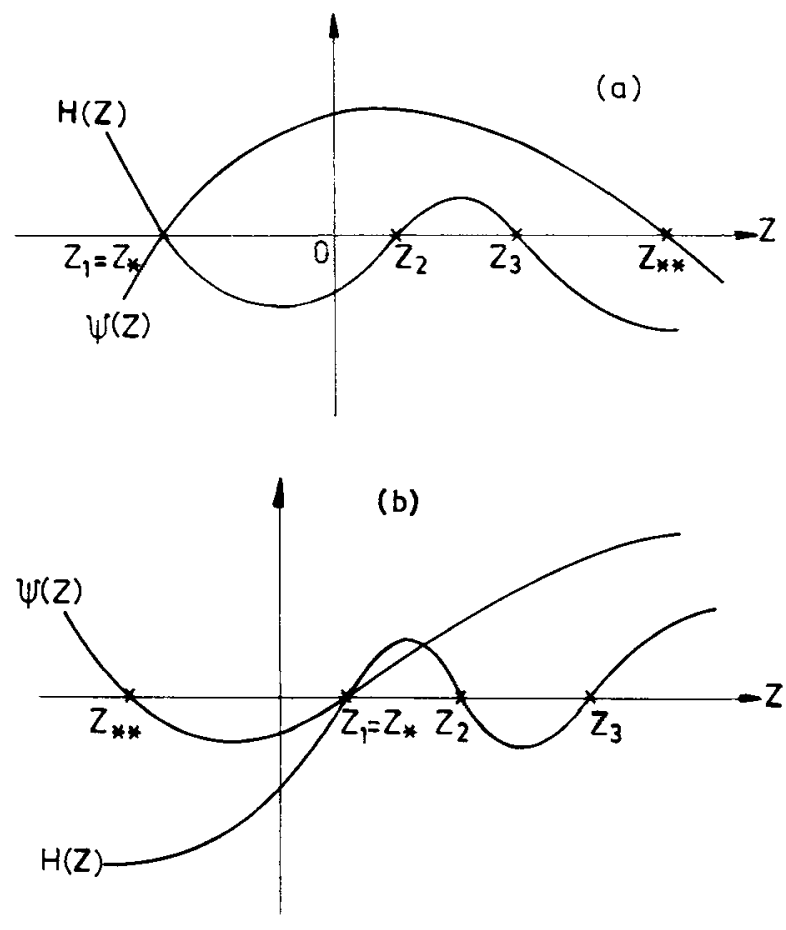

Figure 4. Velocity curves for $\psi(z)$ and $H(z)$ for $z_{1}=z_{*^{*}}$ (a) $m>0$, (b) $m<0, E<|F| \sigma \mid$. 
อ

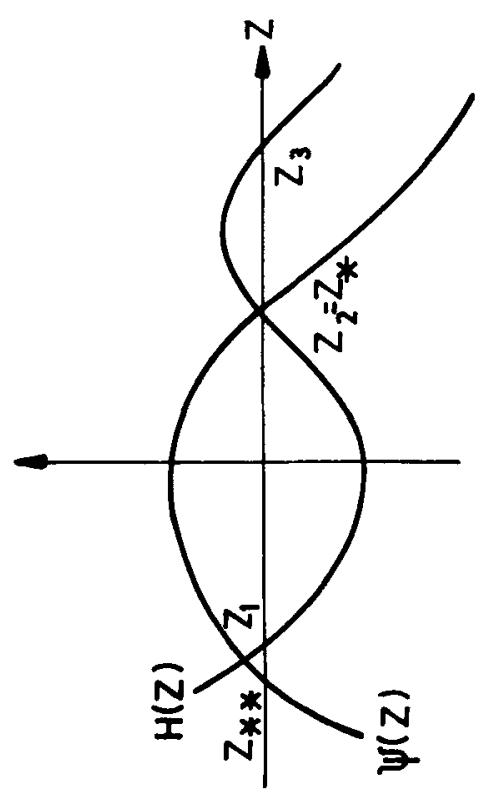

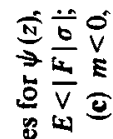

占

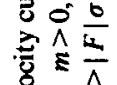

>실

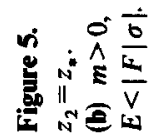

อ
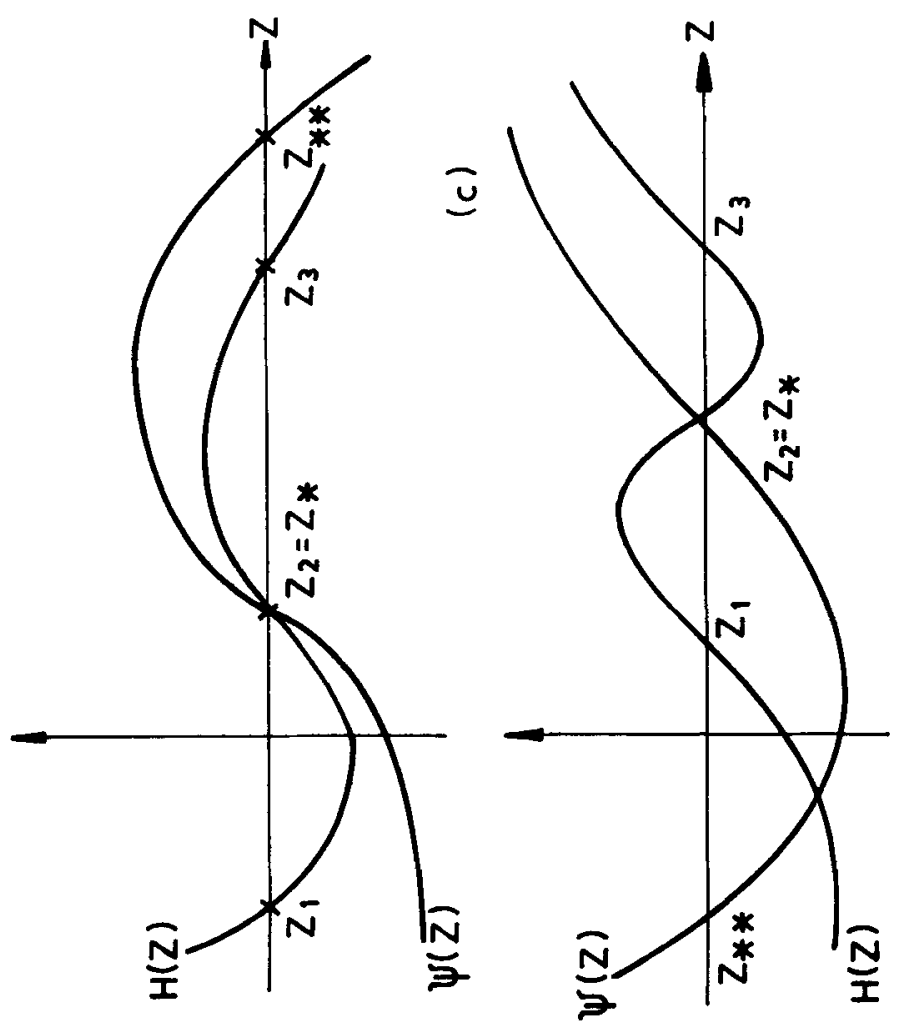
Free flight dynamics of a spinning projectile
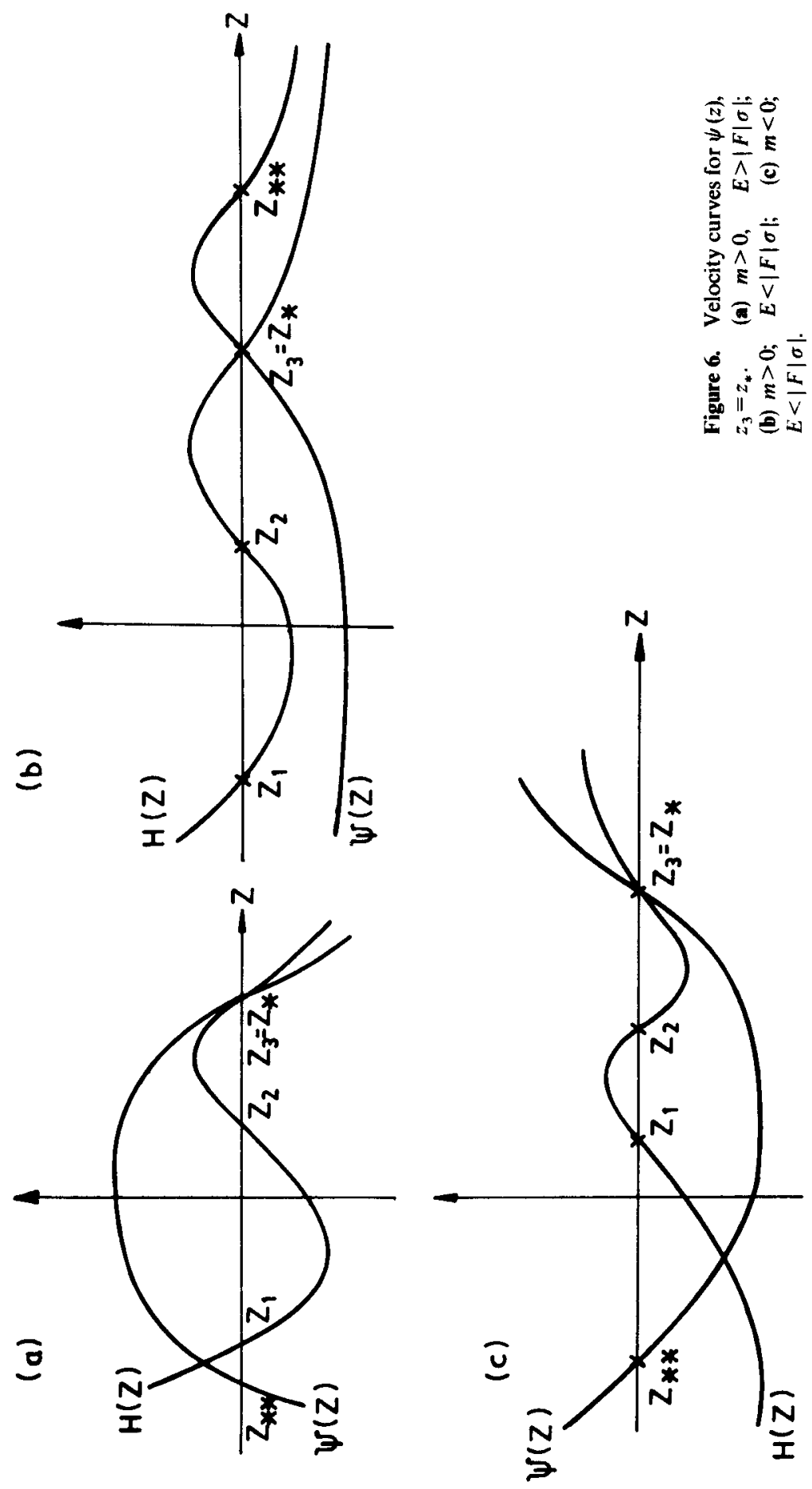


\subsection{Geometry of steady precession}

The invariance of $\phi^{\prime}$ with $\bar{x}$ is steady precession, $\phi^{\prime}=n K_{2}$, where $n$ is a real number. Equation (15a) implies conical motion $(Z=$ constant) of the projectile. In particular $n \rightarrow \frac{1}{2}$ implies that the projectile will topple over by $\pi / 2$. For a large precession $(n \rightarrow \infty)$ the projectile behaves as a gyrostat, pointing along the velocity.

If the conical motion is given by $Z=Z_{2}$, we have

$$
\begin{aligned}
H\left(Z_{2}\right) & \equiv 0, \\
H^{\prime}\left(Z_{2}\right) & \equiv 0 .
\end{aligned}
$$

These equations result in

$$
n^{2}-n+\frac{1}{4}\left[1-\sigma^{2}\left(2 m Z_{2}+1\right)\right]=0 .
$$

Corollary VIII: For $m=0$, the quadratic in $n$ assumes both positive roots $n_{1,2}=(1 \pm \sigma) / 2$. The linear projectile will assume right-handed precession for these two positive values of $n$ for a given $\sigma$.

Corollary IX: For $0<m<\left(1-\sigma^{2}\right) / 2 \sigma^{2} Z_{2}$, (20) gives two positive real roots. The nonlinear projectile can assume any one of these for right-handed steady precession.

For $m>\left(1-\sigma^{2}\right) / 2 \sigma^{2} Z_{2}$, one of the two precessions has to be necessarily lefthanded.

Corollary $X$ : There exists no steady precession for $m<-1 /\left(2 Z_{2}\right)$, while for $-1 /\left(2 Z_{2}\right)<m<\left(1-\sigma^{2}\right) /\left(2 \sigma^{2} Z_{2}\right)$, there exist two positive precessional velocities.

\subsection{Geometry of unsteady precession}

The motion in the $(Z, \phi)$ plane is determined by the equation

$$
\mathrm{d} \phi / \mathrm{d} Z=\left(Z-Z_{*}\right) /\left\{2 \sigma m^{\frac{1}{2}} Z\left[\left(Z-Z_{1}\right)\left(Z-Z_{2}\right)\left(Z_{3}-Z\right)\right]^{\frac{1}{2}}\right\}
$$

from (15a) and (15b). If $\alpha$ is the angle between the motion of (21) and a circle at a point in the plane, then

$$
\tan \alpha=2 \sigma m^{\frac{1}{2}}\left[\left(Z-Z_{1}\right)\left(Z-Z_{2}\right)\left(Z_{3}-Z\right)\right]^{\frac{1}{2}} /\left(Z-Z_{*}\right) .
$$

The curvature $x$ of the curve determined from $(21)$ is

$$
\begin{aligned}
x= & {\left[\left(Z-Z_{*}\right)^{3}+4 \sigma^{2}\left(2 Z-Z_{*}\right) H(Z)-2 \sigma^{2} Z\left(Z-Z_{*}\right)(\mathrm{d} H / \mathrm{d} Z)\right] \div } \\
& \div\left\{Z\left[\left(Z-Z_{*}\right)^{2}+4 \sigma^{2} H(Z)\right]^{3 / 2}\right\} .
\end{aligned}
$$

It is further seen from (21) that motion attains extremum values at circles of radii $0, Z_{1}, Z_{2}, Z_{3}$. The nature of the motion at these circles is analysed from the nature of the second derivative

$$
\mathrm{d}^{2} Z / \mathrm{d} \phi^{2}=\left[2 \sigma^{2} Z /\left(Z-Z_{*}\right)^{3}\right]\left[Z\left(Z-Z_{*}\right)(\mathrm{d} H / \mathrm{d} Z)-2 Z_{*} H(Z)\right] .
$$

It is obvious from the above that the origin is a point of inflexion for the motion.

One may easily see from $(15 \mathrm{a}, \mathrm{b})$ that the singular curves of the motion are the circles of no velocity. The condition $H^{\prime}(Z)=0$ implies that the radial acceleration $Z^{\prime \prime}$ vanishes at these points. In addition if these points are also the zeros of $H(Z),(21)$ reveals that these are the circles of inflexion of the motion. Since $Z^{\prime}$ and $Z^{\prime \prime}$ both vanish at these points, the motion is conical $\left(Z_{2}=Z_{3}\right)$ given by

$$
H(Z)=m\left(Z-Z_{2}\right)^{2}\left(Z-Z_{1}\right)
$$




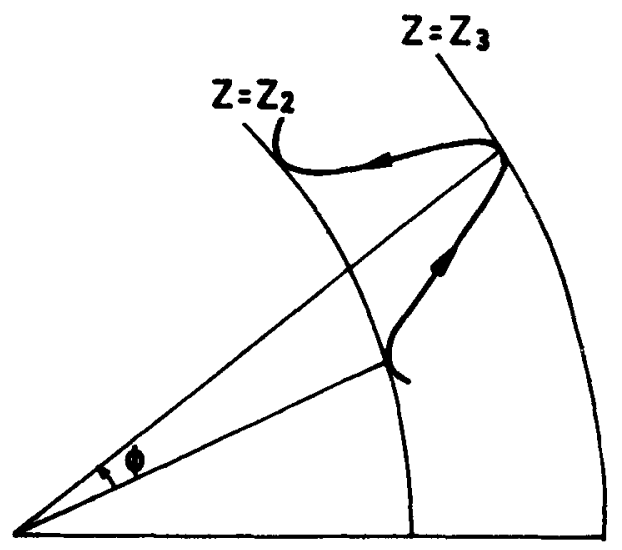

Figure 7. Trajectory for $Z_{*}<Z_{2}$.

We now discuss the motion geometry as the angular momentum parameter $Z_{*}$ travels along the real axis.

Case $I$ : For $-\infty<Z_{*}<Z_{2}$, (21) reveals that $\mathrm{d} \phi>0$ for every $Z$ in $Z_{2}$ to $Z_{3}$ resulting in direct motion. The angle $\alpha[(22)]$ vanishes at the extremum points $Z_{2}$, $Z_{3}$. This proves that the motion is tangential to the circles $Z=Z_{2}, Z_{3}$ (see figure 7). In the case of $Z_{*}=Z_{1}$, the relations (18) reduce to

$$
\begin{aligned}
& Z_{2}+Z_{3}=-(1 / m)-Z_{*}, \\
& Z_{2} Z_{3}=-4 F^{2} / Z_{*} m .
\end{aligned}
$$

That the two roots for real motion are positive implies that

$$
Z_{*}<-1 / m \text {. }
$$

The region for real motion is as shown in figure 8 .

For $Z_{*}=0$, the real motion lies in $0 \leqslant Z \leqslant Z_{3}, Z_{3}=\left[-1+(1+16 E m)^{\frac{1}{2}} / 2 m\right]$. The precession velocity is constant and equals half the spin parameter.

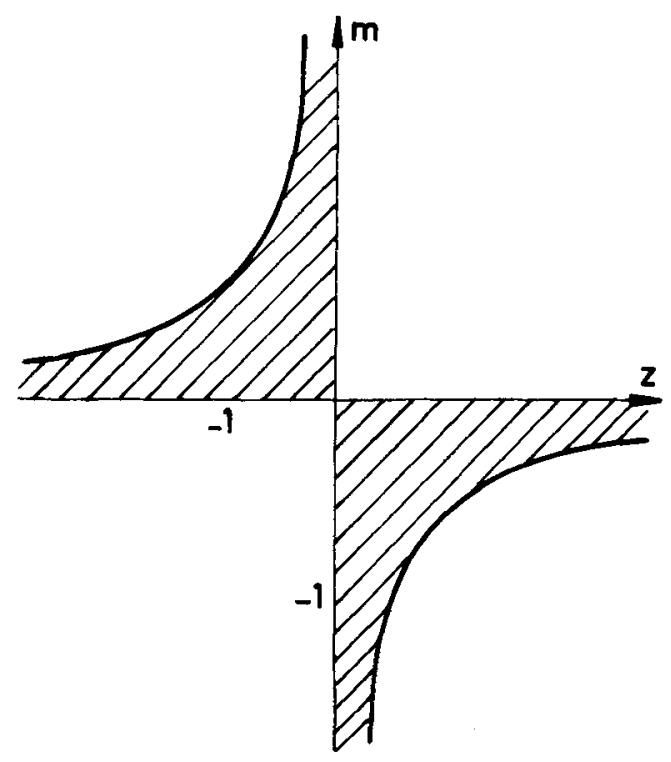

Figure 8. Region of real motion for $Z_{*}=Z_{1}$. 


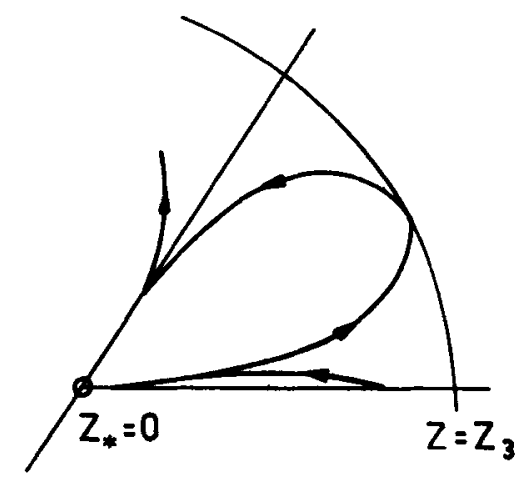

Figure 9. Trajectory for $Z_{*}=0$.

Theorem $I$ : The trajectories at $Z=Z_{2}(=0)$ execute a cuspidal motion for $Z_{*}=0$ (figure 9).

Proof: The curvature of the trajectory (23) tends to infinity as $Z \rightarrow 0$. The motion thus is radial from the origin. The motion is further approximated from (21) in the neighbourhood of this point as

$$
\mathrm{d} \phi / \mathrm{d} Z=A Z^{-\frac{1}{2}},
$$

where

$$
A^{-1}=2 \sigma\left(-m Z_{1} Z_{3}\right)^{\frac{1}{2}}>0
$$

The motion in $(Z, \phi)$ coordinates

$$
\Phi=2 A Z^{\frac{1}{2}},
$$

and in the Cartesian system

$$
\begin{aligned}
Y / x & =\tan 2 A\left(x^{2}+y^{2}\right)^{\frac{4}{4}}, \\
Y^{4} & =16 A^{4} x^{4}\left(x^{2}+y^{2}\right) .
\end{aligned}
$$

The tangent at the origin to the above curve is obtained by equating the lowest degree term to zero,

$$
Y^{4}=0 .
$$

Thus we have a cusp at the origin of a fourth-order contact.

Case II: For $Z_{*}>Z_{3}, \mathrm{~d} \phi<0,(21)$, revealing that the precession of the projectile is negative due to a large initial angular momentum which is also negative. The motion at the extremum points $Z_{2}, Z_{3}$ is also tangential to the circles at these points (see figure 10).

Case III-theorem II: For $Z_{*}=Z_{2}, Z_{3}$, there exists a cuspidal motion at the corresponding circle $Z=Z_{2}, Z_{3}$ (see figures $11 \mathrm{a}, \mathrm{b}$ ). The proof is similar to theorem $I$. The precessional advance $\mathrm{d} \phi>0$ at $Z_{*}=Z_{2}$ while $\mathrm{d} \phi<0$ at $Z_{*}=Z_{3}$. The motion at the other extremum point is tangential to its circle. The cusp appears at the circle of no velocity.

Case $I V$ : For $Z_{2}<Z_{*}<Z_{3}$, a specific value $Z_{*}=Z_{* *}$ is determined from the integral of (21) for which the total travel $\Phi=\phi_{3}-\phi_{2}$ is zero. For this value of $Z_{*}=Z_{* *}$, the path is a closed curve having common tangents with the extremums of the motion (figure 12a). In the case $Z_{2}<Z_{*}<Z_{* *}<Z_{3}$, the total travel is positive, while for 


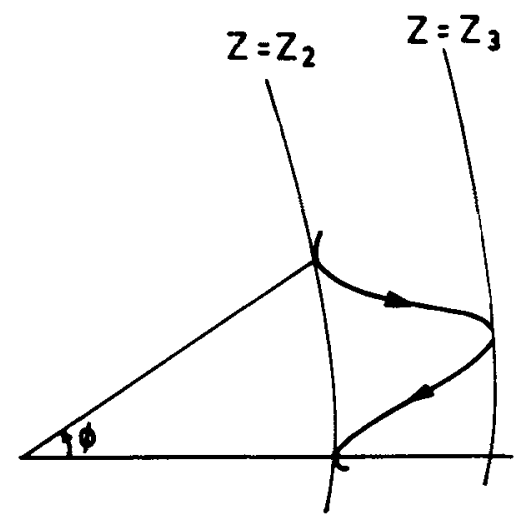

Figure 10. Trajectory for $Z_{*}>Z_{3}$.

$Z_{2}<Z_{* *}<Z_{*}<Z_{3}$, the total travel is negative. From (21), on substituting for $Z_{*}=Z_{* *}+\varepsilon$, we obtain

$$
\Phi=-\varepsilon \int_{Z_{2}}^{Z_{3}} \mathrm{~d} Z /\left\{\sigma Z[H(Z)]^{\frac{1}{2}}\right\}
$$

which is $\gtrless 0$ for $\varepsilon \gtrless 0$.

It is also seen from (21) that $\mathrm{d} \phi<0$ for $Z_{2}<Z<Z_{*}$ and $\mathrm{d} \phi>0$ for $Z_{*}<Z<Z_{3}$. From the above, it is easily deduced that for a complete cycle, a trajectory intersects between $Z_{*}$ and $Z_{3}$ for $Z_{*}<Z_{* *}$, while it intersects between $Z_{*}$ and $Z_{2}$ for $Z_{*}>Z_{* *}$ (figures $12 b, c)$.

\section{Conclusions}

While the equations of free flight motion suggested by Fowler et al (1920), Murphy (1953, 1963) and Nielson \& Synge (1946) do not completely identify the dynamical effects of frame motion on inertia and aerodynamic forces in one form or the other, they have been clearly analysed in $\S 2$ of this paper. Equations (7) completely specify this motion. The ballistic forms of equations have been developed later and are given in (8) and (12).
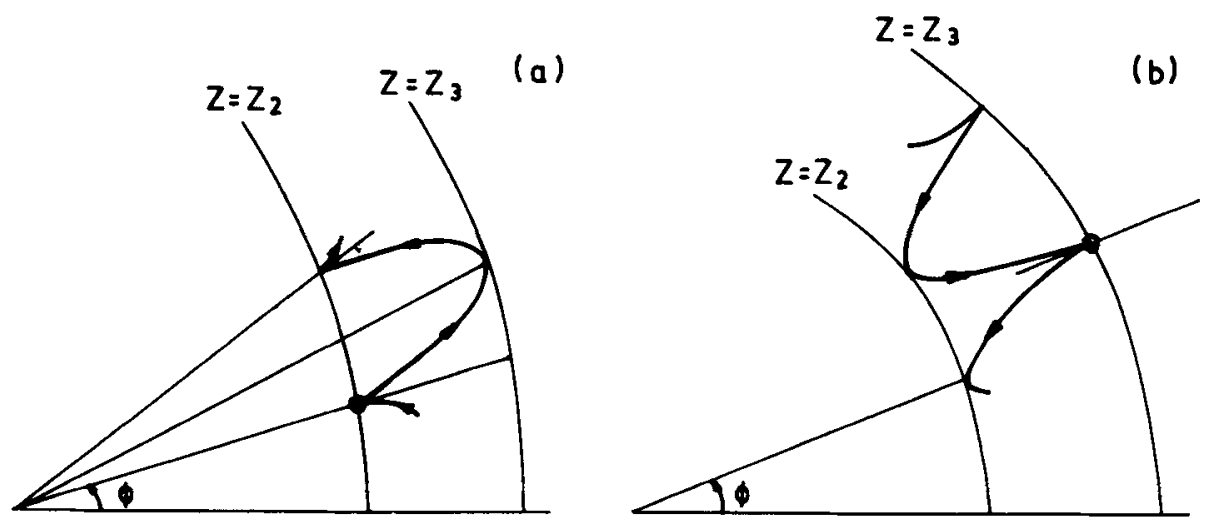

Figure 11. Trajectories for (a) $Z_{*}=Z_{2}$, (b) $Z_{*}=Z_{3}$. 
(a)
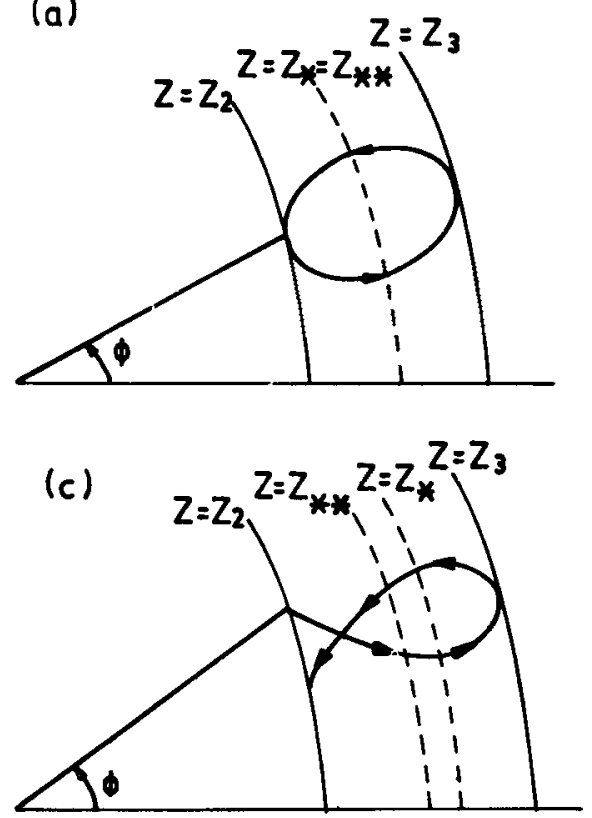

(b)

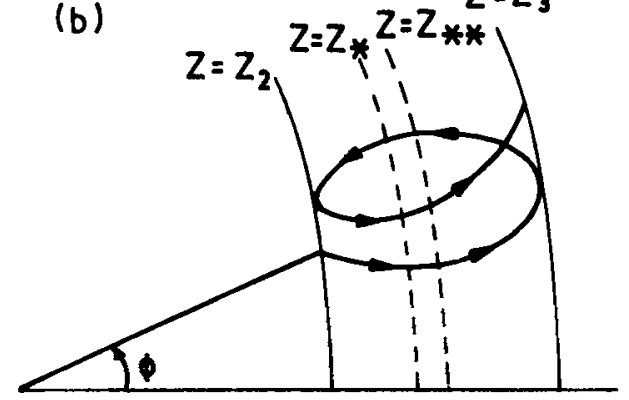

Figure 12. Trajectories for (a) $Z_{2}<Z_{*}=Z_{* *}<Z_{3}$, (b) $Z_{2}<Z_{*}<Z_{* *}<Z_{3}$, (c) $Z_{2}<Z_{* *}<Z_{*}<Z_{3}$.

In $\S 3$ it is proved that the trajectory in the cross-flow plane advances for $Z_{*}<Z_{* *}$ and retrogrades for $Z_{*}>Z_{* *}$. The cusp appears in the motion for $Z_{*}=0, Z_{2}, Z_{3}$, at the corresponding circles $Z=0, Z_{2}, Z_{3}$. Otherwise there exist common tangents to the trajectory and the circle.

The authors are grateful to Prof P C Rath for fruitful discussions, and the Dean \& Director of the Institute for his encouragement. The authors also thank one of the referees for excellent comments and suggestions.

\section{List of symbols}

$\begin{array}{ll}E & \text { energy constant of the motion; } \\ F & \text { angular momentum constant of the motion; } \\ K_{2} & \text { spin parameter; } \\ K_{3} & \text { conservative force parameter; } \\ \bar{V}(u, v, w) & \text { velocity of the projectile; } \\ \bar{\omega}(p, q, r) & \text { angular velocity of the projectile; } \\ \bar{\Omega}\left(\Omega_{x}, \Omega_{y}, \Omega_{z}\right) & \text { angular velocity of the PS frame; } \\ \xi & (v+i w) / u ; \\ \eta & (q+i r) l / u ; \\ \sigma^{2} & 1+\left(4 K_{30} / K_{2}^{2}\right) \text { (Cranz stability parameter). }\end{array}$

\section{Suffixes}

variables in PS frame;

$b$

variables in body frame. 


\section{References}

Etkin B 1971 Dynamics of atmospheric flight (New York: John Wiley and Sons)

Fowler R H, Gallop E G, Lock C N H, Richmand H W 1920 Philos. Trans. R. Soc. A221: 295-387

Maple C G, Synge J L 1949 Q. Appl. Maths. 6: 345-366

Murphy C H 1953 On stability criteria of the Kelley-McShane linearised theory of yawing motion, BRL Rep. no. 853

Murphy C H 1963 Free flight motion of symmetric missiles, BRL Rep. no. 1216

Nieison K L, Synge J L 1946 Q. Appl. Maths. 4: 201-206 